\title{
Analytical Transmission Electron Microscopy Investigation of the Fluorination Process of Carbon Nanoparticles.
}

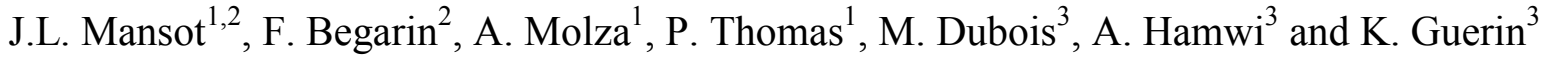 \\ ${ }^{1}$ Groupe de Technologie des Surfaces et des Interfaces (GTSI EA 2432) \\ ${ }^{2}$ Centre Commun de Caractérisation des Matériaux des Antilles et de la Guyane, Université des Antilles \\ et de la Guyane, Campus Fouillole, BP 25097157 Pointe à Pitre - Guadeloupe-France-French West \\ Indies \\ ${ }^{3}$ Clermont Université, Université Blaise Pascal, Institut de Chimie de Clermont-Ferrand (ICCF, UMR \\ 6296), Inorganic Materials team, 63177 Aubière, France
}

Carbon nanoparticles such as graphite nanopowder, exfoliated graphite, carbon nanofibers... are used in several industrial applications such as electrochemistry (lithium batteries) and lubrication [1]. Recent works revealed that the partial or total fluorination significantly improve their properties. However in order to optimize their efficiency and to better monitor the fluorination process, some needs of structural and chemical investigations at nanometric scale are needed in order to characterize the fluorination process and the resulting fluorinated carbon structure. The present study is concerned with the analytical transmission electron microcopy investigation of the structure and composition of partially fluorinated carbon nanoparticles. The results allow us to deduce the fluorination mechanisms involved and to correlate the structure of the fluorinated carbon derivatives to their tribologic performances [2].

Graphitized Carbon nanofibers (MER Corporation) and Carbon blacks (Superior Graphite) are fluorinated by direct fluorination under pure fluorine gas flow in a Monel reactor at temperatures ranging between 380 and $550^{\circ} \mathrm{C}$ in order to obtain atomic $\mathrm{F} / \mathrm{C}$ ratio in the range $0<\mathrm{F} / \mathrm{C}<1$, the $\mathrm{F} / \mathrm{C}$ being determined by ${ }^{19} \mathrm{~F}$ solid NMR and weight uptake [3] .

For carbon blacks derivatives TEM investigations, the particles are simply deposited onto a holey carbon film. For carbon nanofibers fluorinated derivatives a small amount of fibres $(2 \mathrm{mg})$ is embedded into epoxy resin. For TEM characterization, ultrathin sections (60 nm thick) of the epoxy/fibres block are prepared using ultramicrotomy (Leica Ultracut). SEM studies are carried out in low vacuum (130 $\mathrm{Pa}$ ) conditions on a FEI Quanta $250 \mathrm{ESEM}$ equipped with a tungsten cathode running at $5 \mathrm{kV}$. TEM investigations of the structure and composition of the fluorinated carbon nanoparticles are carried out using a FEI Tecnaï F 20 X twin running at $200 \mathrm{kV}$ fitted with an EDAX SiLi detector and a post column Gatan Quantum energy filter.

Typical images and EDS spectra recorded on the two families of carbon nanoparticles and derivatives are presented in figure 1 for different $\mathrm{F} / \mathrm{C}$ ratios. As it can be seen in the case of the two families, at low fluorination rate, only few external layers are affected by the fluorination process. As far as fluorination progresses, the surrounding fluorinated layer thickness increases the internal core of the particles remaining graphitized up to a $\mathrm{F} / \mathrm{C} \approx 0.7$. The measurement of the inter-reticular distances in the fluorinated layer and indexation of the electron micro diffraction patterns allow us to conclude that the fluorinated structure obtained in the case of carbon blacks is $(\mathrm{CF})_{\mathrm{n}}$ whatever the fluorination rate is. For $\mathrm{F} / \mathrm{C}$ ratios $>0.8$ fluorination operates up to the center of the particles. An over fluorination process is also pointed out by the damages generated on the outer part of the particles leading to partial or total delamination of the surrounding layers of the particles. 
The study of structure and composition of fluorinated carbon nanoparticles allowed us to visualize the progression of the fluorination process. The closed structure of both carbon nanofibres and carbon blacks leads to a reduced diffusion of fluorine molecules in the pristine graphite structure limiting the fluorination to the carbon outer shell at low fluorination rate (low temperature). The increase of the reaction temperature increases the diffusion of fluorine and the fluorination process progress toward the inner core (increase of the fluorinated layer). The structural damages generated by the over fluorination of the external layer contribute also to the increase of fluorine diffusion to the pristine core.
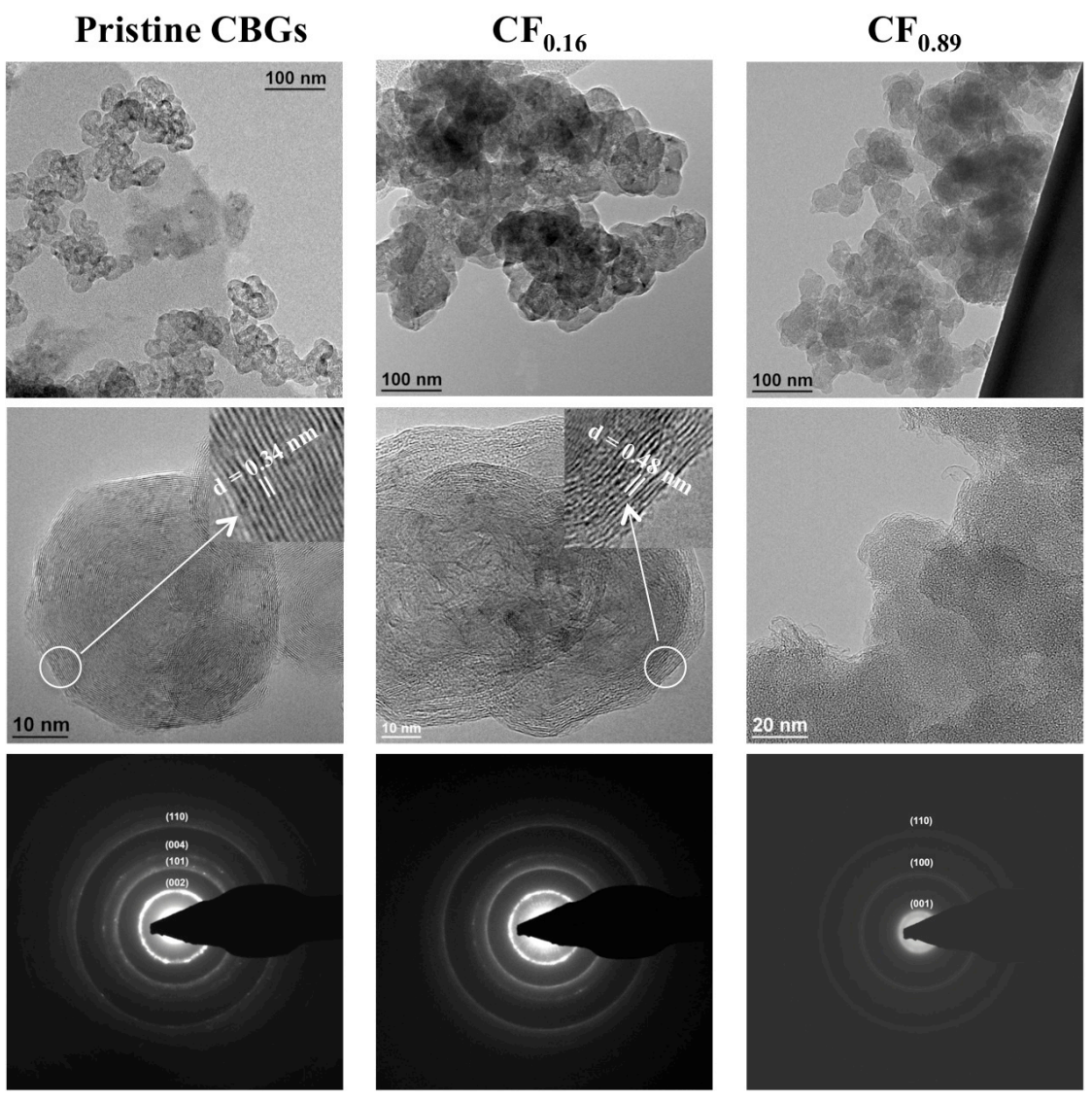

Figure 1. Transmission electron micrographs and electron diffractions patterns collected on pure carbon black (CBGs) and two fluorinated derivatives with $\mathrm{F} / \mathrm{C}$ of 0.16 and 0.89 respectively.

\section{References:}

[1] J.L. Mansot, J.M. Martin, « Colloidal lubricants » in Nanolubricants, Martin J. M., Ohmae O. ed., WILEY New York, (2008).

[2] P. Thomas et al., Tribological properties of fluorinated carbon nanofibres, Tribology Letters 34 (2009), p. 49.

[3] W. Zhang et al.,Fluorinated nanocarbons using fluorinating agent: Strategies of fluorination and application, Eur. Phys. J. (2010) B 75 (2), p. 133.

[4] The authors acknowledge the Ministère de la Recherche, the Conseil Régional de la Guadeloupe, the Conseil Régional de la Martinique, the Fonds Social Européen (FSE) and the Fonds Européens de Développement Régional (FEDER) for their financial supports. 